\title{
A coaxial cable magnetic field sensor based on ferrofluid filled Fabry- Perot interferometer structure
}

\author{
Baokai Cheng, Lei Yuan, Wenge Zhu, Yang Song, Hai Xiao * \\ Department of Electrical and Computer Engineering, Center for Optical Material Science and Engineering Technologies (COMSET), Clemson University, \\ Clemson, SC 29634, USA \\ Received 1 xxxx 2xxx; accepted 15 xxxx $2 x x x$
}

\begin{abstract}
A ferrofluid filled coaxial cable Fabry-Perot interferometer is proposed for magnetic field sensing. Both the interferogram shift and the reflection intensity change of the sensor response are studied when external magnetic field is applied. The sensor is tested to work from 0 Gauss to 160 Gauss, and has a highly linear response above 50 Gauss, with the sensitivity of $459 \mathrm{kHz} / \mathrm{Gauss}$. This sensor can be integrated into a coaxial cable sensing platform for multi-function or distributed sensing applications.
\end{abstract}

Keywords: magnetic field sensor, coaxial cable, interferometer

* Corresponding author. E-mail: haix@clemson.edu

(C) 2016. This manuscript version is made available under the Elsevier user license http://www.elsevier.com/open-access/userlicense/1.0/ 


\section{Introduction}

Ferrofluid is a type of stable colloidal suspension with single domain magnetic nanoparticles that dispersed uniformly in a liquid carrier[1,2]. When exposed to external magnetic field, the nanoparticles inside the ferrofluid will be rearranged, resulting in properties change of the solution such as refractive index and absorption coefficient of electromagnetic radiation[3]. Based on its unique properties, a lot of magnetic field sensors have been proposed. Among them, optical fiber based ferrofluid sensors have attracted a lot of interests because of the advantage of compact size and high sensitivity. Various configurations of fiber optics ferrofluid magnetic field sensor has been reported, such as singlemode-multimodesinglemode (SMS) structure[4], tapered microstructed optical fiber[5], asymmetric optical fiber taper[6], and fiber Fabry-Perot interferometers[7], etc.

Recently, we reported the use of coaxial cable for sensor development. As a low loss waveguide that transmit radio frequency electromagnetic waves, coaxial cables have many similarities to optical fibers, because they share the same electromagnetic cylindrical waveguide theory. Hence, a lot of fiber optics based devices can be migrated on coaxial cables, such as coaxial cable Bragg gratings[8], coaxial cable Fabry-Perot interferometers (FPI)[9]. Sensors such as temperature[10], strain[11] and torsion sensors[12] have also been developed. Physically, coaxial cables are more robust than fibers, making them more suitable for large strain and heavy-duty applications. Different from optical signal, the phase of microwave signals that transmit on the coaxial cables can be easily obtained from commercially available equipment, such as vector network analyser (VNA). With the help of Fourier transform, people can obtain and manipulate both the time domain and frequency domain microwave signal, which offers distributed sensing capability to coaxial cable sensing platform[13].

In this paper, we propose a ferrofluid filled coaxial cable FPI sensor for magnetic field measurement. This sensor is capable of measuring static magnetic field, and has a linear response between 50 Gauss to 160 Gauss, with the sensitivity of $459 \mathrm{kHz} / \mathrm{Gauss}$. Integrated on the coaxial cable sensing platform, it will be a good sensor head for remote and distributed magnetic field sensing applications.

\section{Working principle}

The structure of the sensor is shown in Figure 1. A coaxial cable consists of the inner conductor, outer conductor and the dielectric layer. By removing a small section of dielectric layer in the cable, an air cavity is created inside the cable. A small hole is drilled on the top of the outer conductor, which is used to fill in the ferrofluid, then sealed with tapes. After filling, the interface of the dielectric material and the ferrofluid will act as reflectors, forming a Fabry-Perot (F-P) cavity. Suppose that the reflection coefficient at the two interfaces are equal, when a microwave signal is launched into the cable, the reflected signal at the two interfaces can be expressed as,

$U_{1}=\Gamma \cos [2 \pi f t), \quad U_{2}=\Gamma \cos [2 \pi f(t+\tau)], \quad$ and $\tau=\frac{2 d \sqrt{\varepsilon_{r}}}{c}$

where $\Gamma$ is the reflection coefficient of the interfaces, $f$ is the microwave frequency, $\tau$ is the time delay between the two interfaces of the Fabry-Perot cavity, $d$ is the length of the cavity, $\varepsilon_{r}$ is the effective dielectric constant of the ferro-fluid filled cavity.

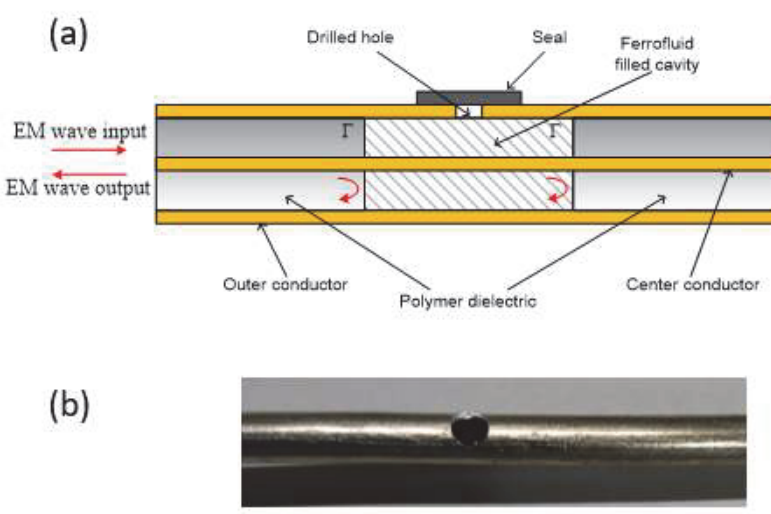

Figure 1. (a) Schematic of the ferrofluid filled coaxial cable sensor. (b) Picture of the sensor (before sealing).

The total reflection signal is the superposition of the 2 reflected signal from the two interfaces of the Fabry-Perot cavity, and can be expressed as,

$U=2 \cdot \Gamma \cos (\pi f \tau) \cos [2 \pi f(t+\tau / 2)]$

This means that the amplitude and the phase of the reflected signal are related to the frequency of the microwave $(f)$ as well as the time delay of the Fabry-Perot cavity $(\tau)$. The spectrum of the reflected signal will exhibit sinusoidal wave in amplitude as an interference pattern, and frequencies of the interference peaks/valleys are,

$$
f_{N}=\frac{N}{\tau}=\frac{N c}{2 d \sqrt{\varepsilon_{r}}} \text {, where } N=1,2,3, \ldots
$$

When the spectrum shifts due to the cavity property change, the relative frequency shift is

$$
\frac{\Delta f_{N}}{f_{N}}=-\frac{\Delta d}{d}-\frac{1}{2} \frac{\Delta \varepsilon_{r}}{\varepsilon_{r}}
$$

Because $\varepsilon_{r}$ of the ferrofluid is related to external magnetic field $B$, when an external magnetic field is applied, the relative frequency shift of the $N$ th peak/valley is

$\frac{\Delta f_{N}}{f_{N}}=-\frac{1}{2} \frac{\Delta \varepsilon_{r}(B)}{\varepsilon_{r}(B)}=-\frac{\Delta n}{n}$

Where $n$ is the effective refractive index of the cavity.

\section{Experiment}

A semi-rigid cable (CrossRF SR-250) is used to fabricate the ferrofluid filled coaxial cable Fabry-Perot interferometer. The outer and inner diameter of the outer conductor are $6.35 \mathrm{~mm}$ and $5.31 \mathrm{~mm}$ respectively, and the diameter of the center conductor is $1.628 \mathrm{~mm}$. The dielectric material is PTFE. We first pull the outer 
conductor away from the semi-rigid cable. A section of the polymer dielectric material of about $7 \mathrm{~cm}$ is cut away from the cable After that, we put the outer conductor back, and an air cavity is created. At the center of the air cavity, a hole of about $2 \mathrm{~mm}$ is drilled on the outer conductor. The ferrofluid (FerroTec EMG909) is filled into the cavity by syringe. After the filling, the hole is sealed with multiple layers of tapes to prevent leakage. This sensor is then connected to the port 1 of a vector network analyzer (VNA). After reading out the S11 information both in magnitude and phase, a complex inverse Fourier transform is performed and we can obtain the time domain reflection (TDR) signal. On the VNA, the frequency span is set from $6 \mathrm{GHz}$ to $12 \mathrm{GHz}$, the IF bandwidth is set to $5 \mathrm{kHz}$, and the total sampling points is 16001 .

The TDR signal of the sensor before and after filling the ferrofluid is shown on figure 2(a). The blue curve is the TDR before filling. The two reflections of the Fabry-Perot cavity are at time of $2.50 \mathrm{~ns}$, and $2.95 \mathrm{~ns}$ with the reflection coefficient of 0.20109 and 0.14247 . The third reflection at $5.54 \mathrm{~ns}$ is caused by the open end of the cable.

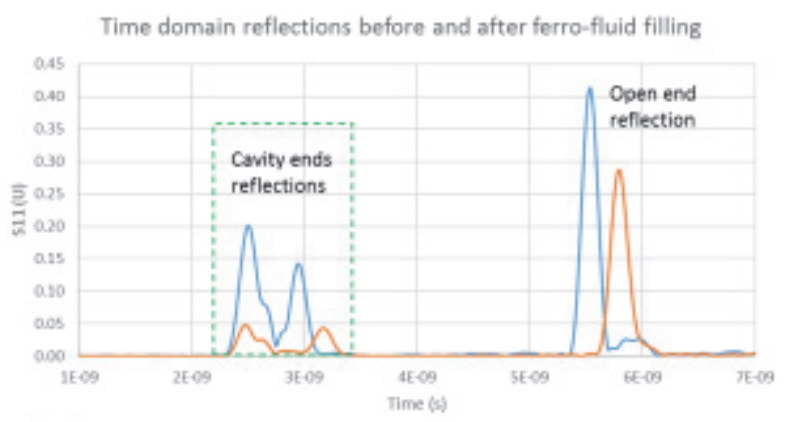

(a)

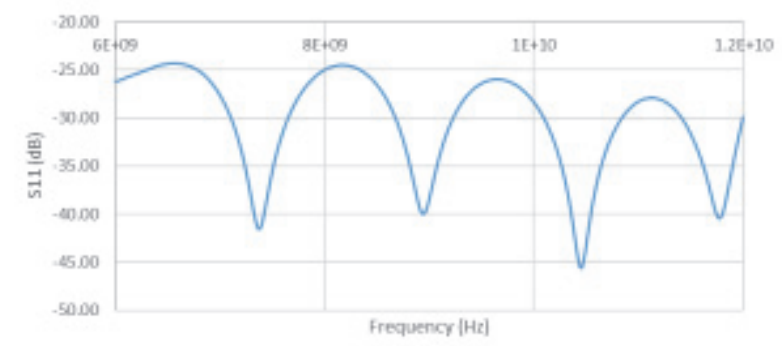

(b)

Figure 2. (a)Time domain reflection signal of the sensor, and (b)Reconstructed interference spectrum of the Fabry-Perot cavity.

The red curve is the TDR after filling the ferrofluid into the cavity. The two reflections of the Fabry-Perot cavity are at time of $2.48 \mathrm{~ns}$, and $3.17 \mathrm{~ns}$ with the reflection coefficient of 0.04788 and 0.04312 . The new open end reflection peak is at $5.79 \mathrm{~ns}$ with reflection coeffeicition of 0.28714. After the ferrofluid filling, the reflections decreased from about $20.1 \%$ and $14.2 \%$ to $4.8 \%$ and $4.3 \%$ respectively. The reflection on the open end also drop from about $41.3 \%$ to $28.7 \%$. There are two reasons for the decrease of the reflection intensity. The first reason is the decrease in refractive index contrast. The reflection coefficient between two sections of coaxial cables is determined by the contrast of the characteristic impedance, which is in turn in proportion to the contrast in refractive index. Before filling the ferrofluid, the contrast is between the cable material and air. After filling, the contrast is between the cable material and the ferrofluid, which is much smaller. This will result in the decrease of reflection intensity at the cavity ends. The second reason is that the ferrofluid will introduce some transmission loss, which will result in reflection intensity drop at the interfaces after the cavity. The reflection peak position that comes after the cavity also changed significantly. Comparing to the TDR before filling, the time delay between the two peaks of the cavity interfaces is increased about $0.24 \mathrm{~ns}$, and the open end reflection peak is delayed about 0.25 ns. Since the cavity length keeps the same, this delay change is induced by the effective refractive index of the ferrofluid. From the contrast of the peak position in the time domain, we can see that the filled ferrofluid introduced an effective index of 1.53 .

The next step is to use a gating window to isolate the two reflectors in time domain, suppressing the other reflection signal. This gating window is shown as a green dotted rectangular in figure 2(a). A Fourier transform is then performed on the gated time-domain signal to convert it back to frequency domain. This technique comes from the joint-time-frequency domain demodulation method, which is the method to realize the distributed sensing capability in cascaded coaxial cable Fabry-Perot interferometry. The details of this technique can be found in [13]. After the transformation, the interference spectrum of the Fabrt-Perot cavity is obtained in frequency domain. Because the interfaces of the cavity are not perfectly created reflectors, and the intensity of the two reflections are not balanced, the resulted spectrum is not a pure a sinusoidal wave as in Equation (1), but with a little distortion as shown in Figure 2(b). Despite of the distortion, when the cavity property change, the spectrum will still shift according to Equation (2).

Figure 3 shows the experiment setup and procedure for testing the response of the sensor to external magnetic field. The cable is connected to the VNA, and a bar magnet is positioned near the Fabry-Perot cavity on the cable to create a magnetic field. A Gauss meter is used to read the field strength, and the probe tip is placed close to the Fabry-Perot cavity. By moving the bar magnet's position, different magnetic field strength from about 0 Gausss to 160 Gausss is created near the sensor, and the frequency domain complex S11 signal is recorded at each field strength. The highest magnetic field the sensor works is limited by the saturation magnetization of the ferrofluid, which is 220 Gauss $\pm 10 \%$. 


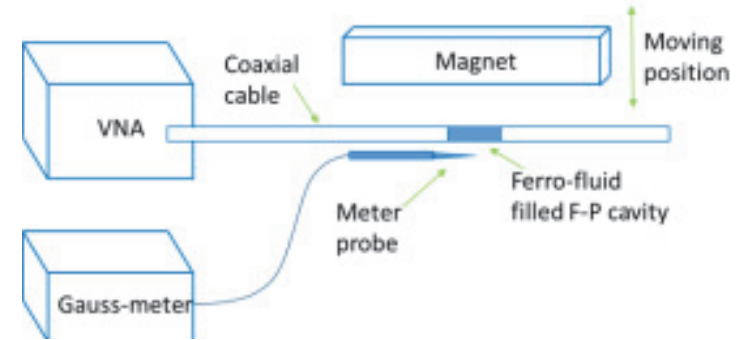

Figure 3. Schematic of the experiment setup for magnetic field measurement.

We first transform the complex S11 signal to time domain and study the change of the peak intensities of the two interfaces of the cavity. In the experiment, the magnetic field is first increased, then decreased. The intensity change is shown in Figure 4.

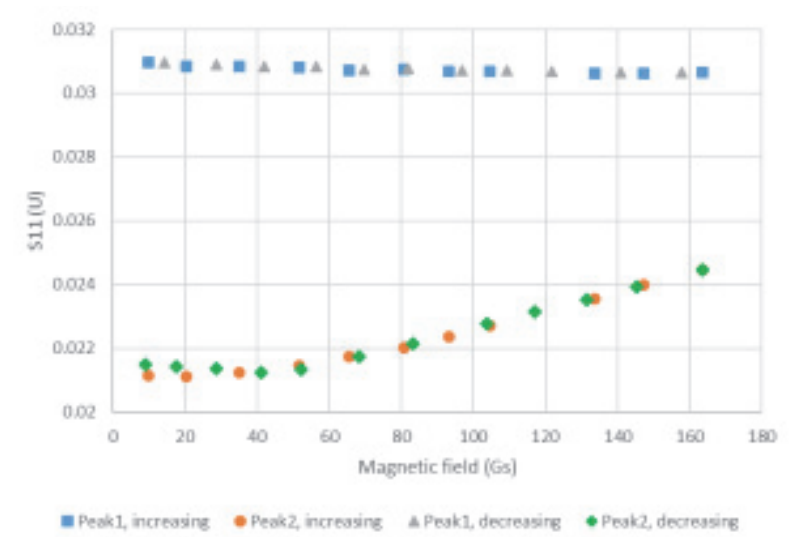

Figure 4 . The reflection intensity of the two cavity interfaces during the process of increasing and decreasing the magnetic field.

From the figure we can see that the reflection intensity of the first interface does not change too much, but the reflection intensity of the second interface increase with the increased magnetic field. There are two possible reasons for the increasing of the intensity: the first is the magnetic field induced refractive index change of the ferrofluid, the second is magnetic field induced microwave absorption change of the ferrofluid. If the dominating reason is the refractive index change, then the intensity from both interfaces should increase, which is not happening. So the intensity change mainly comes from the microwave absorption change. As the external magnetic field increase, the increasing reflection intensity indicates that the microwave absorption is decreased. This is maybe due to the abnormal permeability behaviour in microwave frequency for ferromagnetic nanoparticles[14-16].

From Equation (3) we can see that each dip on the spectrum will respond to the magnetic field, with slight different sensitivity according to the peak frequency. Figure 5 shows the whole spectrum shift when the magnetic field is changed from 0 Gauss to about 160 Gauss. We choose the dip near $9 \mathrm{GHz}$ and plot the spectra near that frequency in Figure 5. As we discussed previously, the intensity of the second reflection increases with the magnetic field, making the intensity of the two reflection more balanced. As a result, the fringe visibility of the interference spectrum also increased from $13 \mathrm{~dB}$ to $17 \mathrm{~dB}$ at the $9 \mathrm{GHz}$ dip. The frequency of the dip is determined by finding the smallest value of the spectrum near $9 \mathrm{GHz}$. To increase the accuracy, curve fitting and interpolation is used. The dip frequency shift with the change of the magnetic field is shown in Figure 6. The magnetic field is first increased from 3 Gauss to 164 Gauss, then decreased to 4 Gauss. The figure shows that from 50 Gauss on, the relationship between the dip frequency and the magnetic field is very linear, and the sensitivity is about $459 \mathrm{kHz} /$ Gauss. Calculated from the data, the refractive index change of the ferrofluid with the magnetic field strength is about $\frac{\Delta f}{f}=$ $-\frac{\Delta n}{n}=0.51 \%$ per 100 Gauss, larger than the $0.3 \%$ change per 100 Gauss value in optical frequency that was reported in [7].

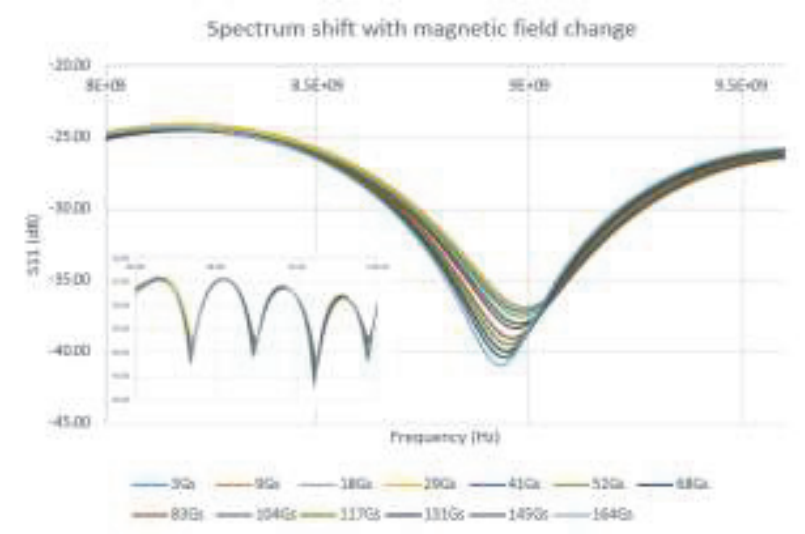

Figure 5. Frequency dip near $9 \mathrm{GHz}$ shifts with magnetic field increase. Inset: Complete spectrum ( $6 \mathrm{GHz}$ to $12 \mathrm{GHz}$ ) shift.

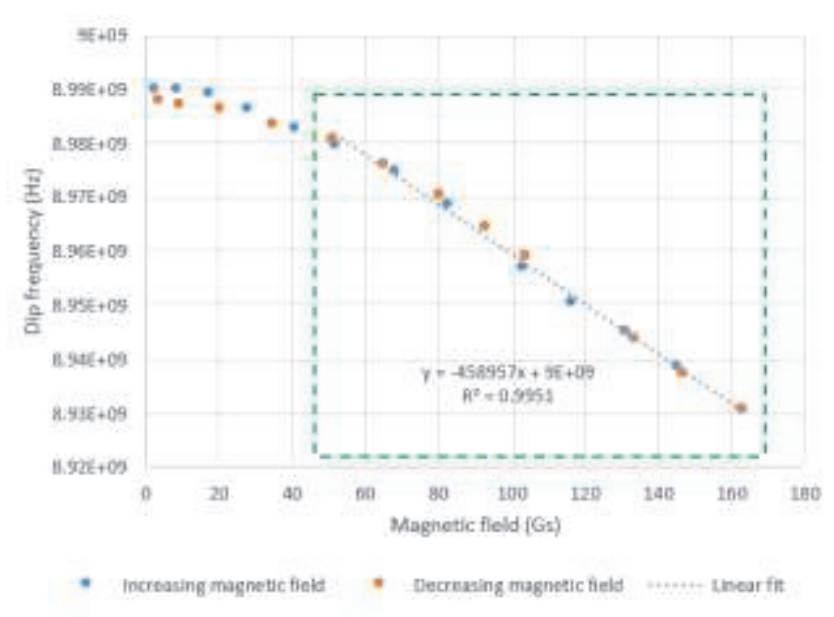

Figure 6. Dip frequency shifts with the process of increasing and decreasing the magnetic field.

In the experiment, the frequency span is $6 \mathrm{GHz}$, and the maximum sampling points from the VNA is 16001 , so the minimum frequency separation between to sampling points is $0.375 \mathrm{MHz}$. However, we can find spectrum dip with higher accuracy by curve fitting and interpolation in the spectrum. From our understanding, the real limitation on the measurement accuracy lies in the stability of the measured spectrum by the VNA, which is influenced by the 
instrument itself. This stability is usually about $10^{-5}$ according to our experience. So the stability of spectrum at about $9 \mathrm{GHz}$ is $90 \mathrm{kHz}$, which is 0.196 Gauss in the magnetic field measurement. Note that the IF bandwidth in the experiment is set to $5 \mathrm{kHz}$, but we cannot achieve that accuracy, because the $90 \mathrm{kHz}$ spectrum stability limit is much larger than it. Temperature crosstalk is also a problem that can influence the sensor performance of the sensor. Temperature variation can change the cavity length with thermal expansion, which induce the $\Delta d$ to occur in Equation (2), and the refractive index of the ferrofluid may also change with temperature. A solution to it is to cascade another Fabry-Perot cavity on the sensor to compensate the thermal expansion part, and the refractive index part can be calculated using RI-temperature coefficient of the ferrofluid, which can be characterized in other experiments.

The proposed magnetic field sensor is based on coaxial cable, which is also a transmission line, making it a good remote sensor. If integrated with other kinds of coaxial cable sensors, multi-function remote sensing can be fulfilled on this platform. If cascaded on a single cable, it also has the potential to realize distributed magnetic field sensing. However, the sensor currently has about $30 \%$ insertion loss, which will limit the number of sensors that can be cascaded. In the future work, we will try to improve the design of the sensor to reduce the loss.

\section{Conclusion}

In conclusion, we propose a ferrofluid filled coaxial cable Fabry-Perot interferometer for magnetic field sensing. The Fabry-Perot cavity is created by cutting away a section of the dielectric material in a semi-rigid coaxial cable, then inject ferro-fluid into it. The interferogram of the Fabry-Perot cavity shifts with the external magnetic field because of the refractive index change of the ferrofluid, and the reflection intensity of the cavity interface also change because of the microwave absorption change. The sensor is tested to work from 0 Gauss to 160 Gauss, and has a highly linear response above 50 Gaus, with sensitivity of $459 \mathrm{kHz} /$ Gauss. The spectrum stability is $90 \mathrm{kHz}$, so the measurement accuracy can be 0.196 Gauss in theory. Based on coaxial cable platform, this magnetic field sensor is good for remote sensing and has the potential for distributed magnetic field sensing applications.

\section{Acknowledgement}

This research was financially supported by the USA Department of Energy (DOE)-National Energy Technology Laboratory (NETL) under grant number DE-FE0009843. dimensional x-ray scattering, Phys. Rev. B. 67 (2003) 094206. S.Y. Yang, Y.F. Chen, H.E. Horng, C.-Y. Hong, W.S. Tse, H.C Yang, Magnetically-modulated refractive index of magnetic fluid films, Appl. Phys. Lett. 81 (2002) 4931.

[4] Y. Chen, Q. Han, T. Liu, X. Lan, H. Xiao, Optical fiber magnetic field sensor based on single-mode-multimode-singlemode structure and magnetic fluid., Opt. Lett. 38 (2013) 39994001.

[5] M. Deng, C. Huang, D. Liu, W. Jin, T. Zhu, All fiber magnetic field sensor with Ferrofluid-filled tapered microstructured optical fiber interferometer., Opt. Express. 23 (2015) 20668-74

[6] M. Deng, D. Liu, D. Li, Magnetic field sensor based on asymmetric optical fiber taper and magnetic fluid, Sensors Actuators A Phys. 211 (2014) 55-59.

[7] R.-Q. Lv, Y. Zhao, D. Wang, Q. Wang, Magnetic Fluid-Filled Optical Fiber Fabry-Pérot Sensor for Magnetic Field Measurement, IEEE Photonics Technol. Lett. 26 (2014) 217 219.

[8] J. Huang, T. Wei, J. Fan, H. Xiao, Coaxial cable Bragg grating assisted microwave coupler, Rev. Sci. Instrum. 85 (2014) 1-5.

[9] J. Huang, T. Wang, L. Hua, J. Fan, H. Xiao, M. Luo, A coaxial cable fabry-perot interferometer for sensing applications., Sensors (Basel). 13 (2013) 15252-60.

[10] A. Trontz, B. Cheng, S. Zeng, H. Xiao, J. Dong, Development of Metal-Ceramic Coaxial Cable Fabry-Pérot Interferometric Sensors for High Temperature Monitoring, Sensors. 15 (2015) 24914-24925.

[11] J. Huang, T. Wei, X. Lan, J. Fan, H. Xiao, Coaxial cable Bragg grating sensors for large strain measurement with high accuracy, Spie. 8345 (2012) 83452Z-83452Z-9.

[12] B. Cheng, W. Zhu, L. Hua, J. Liu, Y. Li, R. Nygaard, H. Xiao, Distributed torsion sensor based on cascaded coaxial cable Fabry-Perot interferometers, Meas. Sci. Technol. 27 (2016) 075103.

[13] J. Huang, X. Lan, W. Zhu, B. Cheng, J. Fan, Z. Zhou, H. Xiao, Interferogram Reconstruction of Cascaded Coaxial Cable FabryPerot Interferometers for Distributed Sensing Application, IEEE Sens. J. 16 (2016) 4495-4500..

[14] C. Mitsumata, S. Tomita, Negative permeability of magnetic nanocomposite films for designing left-handed metamaterials, Appl. Phys. Lett. 91 (2007) 223104.

[15] V.P. Shilov, J.-C. Bacri, F. Gazeau, F. Gendron, R. Perzynski, Y.L. Raikher, Ferromagnetic resonance in ferrite nanoparticles with uniaxial surface anisotropy, J. Appl. Phys. 85 (1999) 6642

[16] Y. Gu, K.G. Kornev, Magnonics: Selective heat production in nanocomposites with different magnetic nanoparticles, J. Appl. Phys. 119 (2016) 095106.

\section{References}

[1] S.W. Charles, The Preparation of Magnetic Fluids, in: Ferrofluids, Springer Berlin Heidelberg, 2002: pp. 3-18.

[2] T. Kruse, H.-G. Krauthäuser, A. Spanoudaki, R. Pelster, Agglomeration and chain formation in ferrofluids: Two- 